WikTOR OKTABA (Lublin)

\title{
NOTE ON THE ANOVA OF A COMPLETELY CONFOUNDED FACTORIAL EXPERIMENT
}

Abstract. The purpose of this paper is to present a modern approach to the analysis of variance (ANOVA) of disconnected resolvable group divisible partially balanced incomplete block (GDPBIB) designs with factorial structure and with some interaction effects completely confounded. A characterization of a factorial experiment with completely confounded interaction is given. The treatment effect estimators and some relations between the matrix $\mathbf{F}$ of the reduced normal equations and the information matrix $\mathbf{A}$ are given. Moreover the ANOVA of the sum of squares for adjusted treatment effects and the matrix $\mathbf{F}$ with its eigenvalues and orthonormal eigenvectors for the case of a completely confounded factorial experiment are presented. A special form of a generalized inverse ( $g$-inverse) of $\mathbf{F}$ is introduced (Theorems 3.2.1-3.2.4). The corresponding numerical example has been worked out by Oktaba (1956) and Oktaba, Rejmak and Warteresiewicz (1956) by applying Galois fields and congruences.

1. Introduction. The literature on confounded factorial experiments is very rich. The classic papers are: Yates $(1933,1937)$ and Fisher (1942). Many statisticians have been interested in the problem of confounding: Bose and Kishen (1940), Finney (1945), Nair (1938), Pearce (1963, 1970, 1976, 1983), C.R. Rao (1951), Cochran and Cox (1950), Kempthorne (1952), Scheffé (1959), Oktaba (1956, 1970, 1971), Caliński and Kageyama (2000, 2003).

In this paper the modern approach to the ANOVA of GD-PIB designs with factorial structure and with some interaction effects completely confounded is presented. The results are given in the theorems. Some of them have special proofs.

2000 Mathematics Subject Classification: Primary 62K10, 62K15.

Key words and phrases: disconnected orthogonal block design, completely confounded design, reduced normal equations, PBIB, Group Divisible (GD), F matrix, Galois field, congruence, ANOVA, graphical method "O". 
The intra-block ANOVA of disconnected incomplete block designs can be based on the method M (Oktaba, 1971), or on the graphical method "O" of constructing independent contrasts (Oktaba, 1958). The corresponding numerical example has been worked out by applying Galois fields and congruences to a real field factorial experiment of the type $4 \times 4 \times 4$ with completely confounded interaction component (Oktaba, 1956; Oktaba et al. 1956).

$\mathbf{1}_{k}$ represents the $k \times 1$ column vector of 1 's, $\mathbf{I}_{k}$ the $k \times k$ identity matrix, $\mathbf{J}_{k}=\mathbf{1}_{k} \mathbf{1}_{k}^{\prime}$ the $k \times k$ matrix of 1 's, $\mathbf{A}$ an $n \times m$ matrix. trace $(\mathbf{A})$ stands for the trace of the square matrix $\mathbf{A} ; \operatorname{rank}(\mathbf{A})$ is the number of non-zero eigenvalues of $\mathbf{A}$, and $|\mathbf{A}|$ denotes its determinant. $\mathbf{A}^{-}$is a $g$-inverse of the matrix $\mathbf{A}$ iff $\mathbf{A} \mathbf{A}^{-} \mathbf{A}=\mathbf{A}$. The Kronecker product of matrices $\mathbf{A}$ and $\mathbf{B}$ is denoted by $\mathbf{A} \otimes \mathbf{B}$.

2. The intrablock ANOVA of a block design $D(t, b, k, r)$, connected or disconnected. A block design is denoted as $D(t, b, k, r)$ if there are $t$ treatments arranged in $b$ blocks, each of size $k$, and every treatment is replicated $r$ times.

A resolvable block design has $t=f k$ treatments (combinations of levels of factors) arranged in $b=f r$ blocks ( $f$ blocks forming one replication) (Street and Street, 1987, Chapter 8).

The intra-block linear model of $n=t r=k b$ observations obtained from the design $D(t, b, k, r)$ can be written as

$$
y_{i j w}=\mu+\tau_{i}+\beta_{j}+e_{i j w}, \quad i=1, \ldots, t ; j=1, \ldots, b ; w=1, \ldots, n_{i j},
$$

where $y_{i j w}$ is the $w$ th observation from the $i$ th treatment in the $j$ th block, $\mu$ is the general mean, $\tau_{i}$ is the $i$ th treatment effect, $\beta_{j}$ is the $j$ th block effect and $e_{i j w}$ 's are residuals distributed identically and independently with

$$
\mathrm{E}\left(e_{i j w}\right)=0, \quad \operatorname{Var}\left(e_{i j k}\right)=\sigma^{2}, \quad \operatorname{Cov}\left(e_{i j w}, e_{i^{\prime} j^{\prime} w^{\prime}}\right)=0
$$

for all $i j w \neq i^{\prime} j^{\prime} w^{\prime}$.

The model (2.1) can be expressed as (Pearce, Caliński and Marshall, 1974)

$$
\underset{n 1}{\mathbf{y}}=\mu \mathbf{1}_{n}+\underset{n t}{\boldsymbol{\Delta}_{t 1}^{\prime}} \boldsymbol{\boldsymbol { \tau }}+\underset{n b}{\mathbf{D}_{b 1}^{\prime}} \boldsymbol{\beta}+\underset{n 1}{\mathbf{e}}=\mathbf{X} \boldsymbol{\theta}+\mathbf{e},
$$

where $\mathbf{e}=\left(e_{1}, \ldots, e_{n}\right)^{\prime}$ is an $n$-component residual vector, having $\mathrm{E}(\mathbf{e})=\mathbf{0}$ and $\operatorname{Cov}(\mathbf{e})=\sigma^{2} \mathbf{I}_{n}, \mathbf{y}=\left(y_{1}, \ldots, y_{n}\right)^{\prime}$ is an $n$-component vector of observations, $\mathbf{X}=\left(\mathbf{1}_{n}, \boldsymbol{\Delta}_{n t}^{\prime}, \mathbf{D}_{n b}^{\prime}\right)$ is the $n \times(1+t+b)$ design matrix partitioned into the $n$-component vector $\mathbf{1}_{n}$, an $n \times t$ design matrix for treatments $\boldsymbol{\Delta}^{\prime}$ and an $n \times b$ design matrix for blocks $\mathbf{D}^{\prime}$, and where $\boldsymbol{\theta}=\left(\mu, \boldsymbol{\tau}^{\prime}, \boldsymbol{\beta}^{\prime}\right)^{\prime}$ is a $(1+b+t)$ component vector with $\boldsymbol{\tau}=\left(\tau_{1}, \ldots, \tau_{t}\right)^{\prime}$ and $\boldsymbol{\beta}=\left(\beta_{1}, \ldots, \beta_{b}\right)^{\prime}$. The elements of the $\boldsymbol{\Delta}$ and $\mathbf{D}$ matrices are 0 or 1 . Let $\mathbf{y} \sim \mathcal{N}_{n}\left(\mathbf{X} \boldsymbol{\theta}, \sigma^{2} \mathbf{I}_{n}\right)$ (i.e., has an 
$n$-variable normal distribution). We also have $\mathbf{T}=\boldsymbol{\Delta} \mathbf{y}=\left(T_{1}, \ldots, T_{t}\right)^{\prime}$, the vector of treatment totals, $\mathbf{B}=\mathbf{D} \mathbf{y}=\left(B_{1}, \ldots, B_{b}\right)^{\prime}$, the vector of block totals, and $\mathbf{1}_{t}^{\prime} \mathbf{T}=\mathbf{1}_{b}^{\prime} \mathbf{B}=\mathbf{1}_{n}^{\prime} \mathbf{y}=G$, the grand total.

The $t \times b$ incidence matrix $\underset{t b}{\mathbf{N}}=\mathbf{\Delta} \mathbf{D}^{\prime}$ has elements $n_{i j}(i=1, \ldots, t ; j=$ $1, \ldots, b)$, where $n_{i j}$ is the number of 1 's in the $j$ th block receiving the $i$ th treatment.

Let

$$
\underset{n n}{\phi}=\mathbf{I}_{n}-\frac{1}{k} \mathbf{D}^{\prime} \mathbf{D}, \quad \operatorname{rank}(\underset{t n}{\boldsymbol{\Delta}} \boldsymbol{\phi n})=h<t .
$$

The normal equations under the constraints

$$
\mathbf{1}_{b}^{\prime} \boldsymbol{\beta}=0 \quad \text { and } \quad \mathbf{1}_{t}^{\prime} \boldsymbol{\tau}=0
$$

are

$$
\left\{\begin{array}{l}
G=n \widehat{\mu}, \quad \mathbf{B}=k \mathbf{1}_{b} \widehat{\mu}+k \widehat{\boldsymbol{\beta}}+\mathbf{N}^{\prime} \widehat{\boldsymbol{\tau}}, \\
\underset{t 1}{\mathbf{T}}=r \mathbf{1}_{t} \widehat{\mu}+\mathbf{N} \widehat{\boldsymbol{\beta}}+r \widehat{\boldsymbol{\tau}} .
\end{array}\right.
$$

The reduced normal equations for studying the treatment effects $\widehat{\boldsymbol{\tau}}$ are

$$
r \underset{t t}{\mathbf{F}} \widehat{\boldsymbol{\tau}}=\underset{t 1}{\mathbf{Q}}
$$

where

$$
\begin{gathered}
\mathbf{F}=\frac{1}{r} \mathbf{A}=\mathbf{I}_{t}-\mathbf{M}, \\
\mathbf{A}=r \mathbf{F}=\boldsymbol{\Delta} \boldsymbol{\phi} \boldsymbol{\Delta}^{\prime}
\end{gathered}
$$

is the information matrix, $\mathbf{N N}^{\prime}$ is the so-called concordance (John, 1980) or concurrence matrix (Pearce, 1963), $\mathbf{M}=\frac{1}{k r} \mathbf{N N}^{\prime}$ is Jones' (1959) M matrix of order $t \times t$, and

$$
\underset{t 1}{\mathbf{Q}}=\mathbf{T}-\frac{1}{k} \mathbf{N B}=\underset{t n}{\boldsymbol{\Delta}} \underset{n n}{\boldsymbol{\phi}} \underset{n 1}{\mathbf{y}}=\left(Q_{1}, \ldots, Q_{t}\right)^{\prime}
$$

is the vector of adjusted treatment totals. The matrix $\phi$ defined in (2.3) has the properties

$$
\phi^{\prime}=\phi, \quad \phi \phi=\phi, \quad \phi \mathbf{1}_{n}=0, \quad \phi \mathbf{D}^{\prime}=0 .
$$

The residual sum of squares is given by

$$
S S_{e}=\mathbf{y}^{\prime} \psi \mathbf{y}
$$

where $\boldsymbol{\psi}=\boldsymbol{\phi}-\boldsymbol{\phi} \boldsymbol{\Delta}^{\prime} \mathbf{A}^{-} \boldsymbol{\Delta} \boldsymbol{\phi}$ (see Caliński and Kageyama, 2000, p. 65).

The term

$$
S S_{\text {tr adj }}=\frac{1}{r} \mathbf{Q}^{\prime} \mathbf{F}^{-} \mathbf{Q}
$$

represents, in the intra-block ANOVA, the treatment sum of squares adjusted for blocks. 
Definition 2.1. An incomplete block design $D(t, b, k, r)$ is disconnected if $\operatorname{rank}(\mathbf{F})=t-f=h$, where $f>1$.

If $f=1$ the design is connected. Then all the elementary contrasts are estimable in the intra-block analysis.

The orthogonal and non-orthogonal experimental designs are discussed in (Oktaba, 2002, pp. 87-89).

The condition $\mathbf{N}=\frac{1}{n} \mathbf{r k}^{\prime}$ for orthogonality between blocks and treatments of a block design is given by Caliński, Ceranka and Mejza (1979, pp. 178-189) who proposed a general definition of an orthogonal block design. Caliński and Kageyama (2000, Sections 2.2, 2.3 and 3.6; 2003, Section 7.2) also discuss the orthogonal designs.

\section{Factorial disconnected incomplete block design with some of interaction/main effects completely confounded with blocks}

3.1. Some methods of analysis. (i) The general method of statistical interpretation of the data obtained in incomplete block designs with effects of interaction (interaction components) completely confounded with blocks has been described by many authors. It is based on congruences and Galois fields. The theory was applied to a block design of the type $4 \times 4 \times 4$ (3 factors at four levels each) with the effects of three interaction components completely confounded with blocks. The factorial experiment was laid down at the Experimental Agricultural Station, Felin, Lublin University of Agriculture (Oktaba, 1956; Oktaba et al., 1956).

(ii) The same experiment can be analysed by a method using the eigenvalues and the eigenvectors of the $\mathbf{F}$ matrix, and the theory of block designs with factorial structure (Nigam et al., 1988, pp. 64-92; Tocher, 1952; Pearce et al., 1974; Caliński, 1983). Estimations of the treatment effects and the sum of squares for adjusted treatments are expressed by formulas including the matrix $\mathbf{F}$. This sum of squares can be obtained by applying the graphical method "O" (Oktaba, 1971). The method M (Oktaba, 1971) is useful in the intra-block ANOVA of a disconnected block design.

3.2. Characterization of a factorial experiment with interaction/main effects completely confounded. Let us consider a block design as in Section 2. It is possible to have a one-to-one correspondence between treatments of a block design and the treatment combinations of a factorial experiment with $r$ blocks involving the same set of treatment combinations. It is assumed that some interaction/main effects are completely confounded with blocks, so that $t=f k$ (cf. Sec. 2).

A factorial experiment with some confounded interaction/main effects is a simple design, so there is no problem with the ANOVA. The design is resolvable, because $b=r f$. The $t=f k$ treatment combinations satisfy 
the following association scheme: the $t$ treatment combinations are divided into $f$ groups (arranged in different blocks) each of size $k$ such that any two combinations of the same group are first associates (they occur together in $\lambda_{1}=r$ blocks) and those of different groups are second associates (they occur together in $\lambda_{2}=0$ blocks).

The eigenvalues of $\mathbf{M}$ are:

$$
\left\{\begin{array}{l}
\mu_{0}=1, \\
\mu_{1}=\frac{\theta_{1}}{r k}=0 \quad \text { with multiplicity } \varrho_{1}=t-f, \\
\mu_{2}=\frac{\theta_{2}}{r k}=\frac{r k}{r k}=1 \quad \text { with multiplicity } \varrho_{2}=f-1,
\end{array}\right.
$$

(Bose and Connor, 1952; Nigam et al., 1988, pp. 33, 86-87; Jones, 1959, p. 176).

From (3.2.1) it follows that the losses of unconfounded effects are equal to zero. There are $h=(t-1)-(f-1)=t-f$ unconfounded (full efficiency) degrees of freedom which correspond to the eigenvalue 0 of the matrix $\mathbf{M}$ with multiplicity $\varrho_{1}=h=t-f$.

Thus, we have only $h=t-f(f>1)$ linearly independent contrasts estimable in the intra-block analysis (full efficiency) and not $t-1$ as in the case of a connected design.

Theorem 3.2.1. In the disconnected design $D(t, b, k, r)$ with a completely confounded component interaction (main effect) the treatment effects $\widehat{\tau}$ are

$$
\widehat{\boldsymbol{\tau}}=\frac{1}{r} \mathbf{F}^{-} \mathbf{Q}=\boldsymbol{\Omega}_{p} \mathbf{Q}=\mathbf{A}^{-} \mathbf{Q},
$$

where

$$
\left\{\begin{array}{l}
\mathbf{F}=\mathbf{I}_{f} \otimes \mathbf{A}_{1}=\mathbf{F}^{-}, \\
\operatorname{rank}(\mathbf{F})=t-f=h, \\
\operatorname{rank}\left(\mathbf{A}_{1}\right)=k-1,
\end{array}\right.
$$

$\mathbf{Q}$ is as in (2.9) and $\boldsymbol{\Omega}_{p} \mathbf{A}^{-}=\frac{1}{r} \mathbf{F}^{-}, \mathbf{A}_{1}=\mathbf{I}_{k}-\frac{1}{k} \mathbf{J}_{k}$.

Proof. The incidence matrix $\mathbf{N}$ is

$$
\underset{t b}{\mathbf{N}}=\underbrace{\left[\begin{array}{l}
\mathbf{L}, \ldots, \mathbf{L} \\
t f
\end{array}\right.}_{r \text { times }}, \quad \underset{t f}{\mathbf{L}}=\mathbf{I}_{f} \otimes \mathbf{1}_{k} .
$$

Then

$$
\mathbf{N N}^{\prime}=[\mathbf{L}, \ldots, \mathbf{L}]\left[\begin{array}{c}
\mathbf{L}^{\prime} \\
\vdots \\
\mathbf{L}^{\prime}
\end{array}\right]=r \mathbf{L} \mathbf{L}^{\prime}=r\left(\mathbf{I}_{f} \otimes \mathbf{J}_{k}\right)=\mathbf{I}_{f} \otimes r \mathbf{J}_{k}
$$


Thus we have

$$
\mathbf{M}=\mathbf{M}^{\prime}=\frac{1}{r k} \mathbf{N N}^{\prime}=\frac{1}{r k}\left(\mathbf{I}_{f} \otimes r \mathbf{J}_{k}\right)=\frac{1}{k}\left(\mathbf{I}_{f} \otimes \mathbf{J}_{k}\right)
$$

and

$$
\operatorname{rank} \mathbf{M}=\operatorname{rank}\left(\mathbf{I}_{f} \otimes \mathbf{J}_{k}\right)=\operatorname{rank} \mathbf{I}_{f} \cdot \operatorname{rank} \mathbf{J}_{k}=f \cdot 1=f .
$$

Since $\mathbf{F}=\mathbf{I}_{t}-\mathbf{M}=\mathbf{I}_{t}-\frac{1}{r k} \mathbf{N N}^{\prime}$ and $t=f k$ we obtain

$$
\mathbf{F}=\mathbf{I}_{f} \otimes \mathbf{A}_{1}, \quad \text { where } \quad \mathbf{A}_{1}=\mathbf{I}_{k}-\frac{1}{k} \mathbf{J}_{k} .
$$

Note that as $\mathbf{F} \mathbf{1}=0$, the matrix $\mathbf{F}$ is singular.

By Lemma 4.3 (Appendix) $\mathbf{A}_{1}=\mathbf{A}_{1}^{2}=\mathbf{A}_{1}^{-}$, so

$$
\mathbf{F}^{-}=\left(\mathbf{I}_{f} \otimes \mathbf{A}_{1}\right)^{-}=\mathbf{I}_{f}^{-} \otimes \mathbf{A}_{1}^{-}=\mathbf{I}_{f} \otimes \mathbf{A}_{1}=\mathbf{F}
$$

and by (2.8),

$$
\mathbf{A}^{-}=(r \mathbf{F})^{-}=\frac{1}{r} \mathbf{F}^{-}=\frac{1}{r}\left(\mathbf{I}_{f} \otimes \mathbf{A}_{1}\right)=\boldsymbol{\Omega}_{p},
$$

where

$$
\boldsymbol{\Omega}_{p}=\frac{1}{r} \mathbf{F}=\frac{1}{r} \mathbf{F}^{-},
$$

$\operatorname{rank}\left(\mathbf{A}^{-}\right)=\operatorname{rank}\left(\mathbf{F}^{-}\right)=\operatorname{rank}(\mathbf{F})=\operatorname{rank}(\mathbf{A})=\operatorname{rank}\left(\mathbf{I}_{f}\right) \cdot \operatorname{rank}\left(\mathbf{A}_{1}\right)$

$$
=f \cdot \operatorname{rank}\left(\mathbf{I}_{k}-\frac{1}{k} \mathbf{J}_{k}\right)=f(k-1)=f k-f=t-f=h \quad \text { (say), }
$$

$\operatorname{rank}\left(\mathbf{A}_{1}\right)=\operatorname{rank}\left(\mathbf{I}_{k}-\frac{1}{k} \mathbf{J}_{k}\right)=k-1 \quad[$ cf. (4.3), Lemma 4.2]

Evidently the vector $\boldsymbol{\tau}$ of treatment parameters is not estimable in the intra-block ANOVA. Of course, some linear functions of $\boldsymbol{\tau}$, say $v^{\prime} \boldsymbol{\tau}$, are estimable (see, e.g., Theorems 4.1 and 4.2 in Caliński, 1983). Note that $\widehat{\tau}$ is simply a solution, nonunique, of the equation (2.6).

TheOREm 3.2.2. The sum of squares for adjusted treatments $S S_{\text {tr adj }}$ in the intra-block ANOVA of a disconnected design $D(t, b, k, r)$ with a component interaction/main effect completely confounded can be expressed in the form

$$
S S_{\mathrm{tr} \text { adj }}=\mathbf{Q}^{\prime} \boldsymbol{\Omega}_{p} \mathbf{Q}=\frac{1}{r} \mathbf{Q}^{\prime} \mathbf{Q}=\frac{1}{r} \sum_{i=1}^{t} T_{i}^{2}-\frac{1}{r k} \sum_{j=1}^{f} H_{j}^{2}
$$

with $\operatorname{rank}(\mathbf{F})=t-f=h$ degrees of freedom, where 


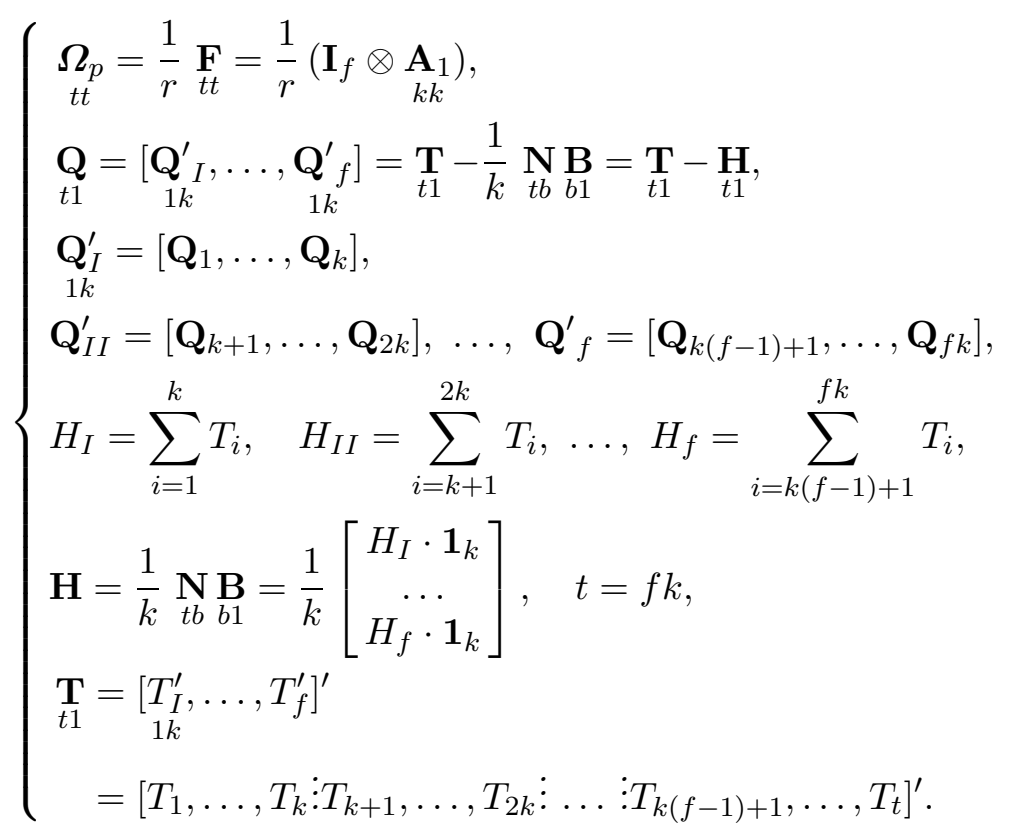

Proof. By (2.12) and (3.2.3) we get $S S_{\mathrm{tr} \text { adj }}=\frac{1}{r} \mathbf{Q}^{\prime} \mathbf{F} \mathbf{Q}$. From

$$
\mathbf{Q}_{k 1}=\mathbf{T}_{k 1}-\frac{1}{k} H_{j} \cdot \mathbf{1}_{k} \quad(j=I, I I, \ldots, f)
$$

and (3.2.7) we have

$$
\begin{aligned}
& S S_{\mathrm{tr} \text { adj }}=\underset{1 t}{\mathbf{Q}^{\prime}} \boldsymbol{\Omega}_{p t} \underset{t 1}{\mathbf{Q}}=\frac{1}{r} \underset{1 k}{\left.\mathbf{Q}_{I}^{\prime}, \ldots, \underset{1 k}{\mathbf{Q}_{f}^{\prime}}\right]\left(\mathbf{I}_{f} \otimes \underset{k k}{\mathbf{A}_{1}}\right)}\left[\begin{array}{c}
\mathbf{Q}_{I} \\
\vdots \\
\mathbf{Q}_{f}
\end{array}\right] \\
& =\frac{1}{r} \underset{1 k}{\left[\mathbf{Q}_{I}^{\prime}, \ldots, \underset{1 k}{\mathbf{Q}_{f}^{\prime}}\right]}\left[\begin{array}{ccc}
\mathbf{A}_{1} & & 0 \\
& \ddots & \\
0 & & \mathbf{A}_{1}
\end{array}\right]\left[\begin{array}{c}
\mathbf{Q}_{I} \\
\vdots \\
\mathbf{Q}_{f}
\end{array}\right] \\
& =\frac{1}{r} \sum_{i=1}^{f} \underset{1 k}{\mathbf{Q}_{i}^{\prime}} \underset{k k}{\mathbf{A}_{1}} \underset{k 1}{\mathbf{Q}_{i}}
\end{aligned}
$$

Since $\mathbf{Q}_{j}^{\prime} \mathbf{J}_{k}=0(j=I, \ldots, f)$, we get

$$
\begin{aligned}
\frac{1}{r} \mathbf{Q}_{I}^{\prime} \mathbf{A}_{1} \mathbf{Q}_{I} & =\frac{1}{r} \mathbf{Q}_{I}^{\prime}\left(\mathbf{I}_{k}-\frac{1}{k} \mathbf{J}_{k}\right) \mathbf{Q}_{I}=\frac{1}{r} \mathbf{Q}_{I}^{\prime} \mathbf{Q}_{I}=\frac{1}{r} \sum_{i=1}^{k} \mathbf{Q}_{i}^{2} \\
& =\frac{1}{r}\left(\mathbf{T}_{I}-\frac{1}{k} H_{I} \cdot \mathbf{1}_{k}\right)^{\prime}\left(\mathbf{T}_{I}-\frac{1}{k} H_{I} \cdot \mathbf{1}_{k}\right)=\frac{1}{r} \sum_{i=1}^{k} T_{i}^{2}-\frac{1}{r k} H_{1}^{2} .
\end{aligned}
$$


Analogously we obtain

$$
\begin{aligned}
\frac{1}{r} \mathbf{Q}_{I I}^{\prime} \mathbf{A}_{1} \mathbf{Q}_{I I} & =\frac{1}{r} \sum_{i=k+1}^{2 k} T_{i}^{2}-\frac{1}{r k} H_{I I}^{2}, \ldots, \\
\frac{1}{r} \mathbf{Q}_{f}^{\prime} \mathbf{A}_{1} \mathbf{Q}_{f} & =\frac{1}{r} \sum_{i=(f-1) k+1}^{f k} T_{i}^{2}-\frac{1}{r k} H_{f}^{2} .
\end{aligned}
$$

Then

$$
S S_{\text {tr adj }}=\frac{1}{r} \sum_{j=1}^{t} T_{i}^{2}-\frac{1}{r k} \sum_{j=1}^{f} H_{j}^{2} .
$$

Note that the proof of Theorem 3.2.2 can be shortened if we use $\mathbf{F}^{-}=\mathbf{I}_{t}$. Moreover: if we take as $\mathbf{F}^{-}$the matrix $\mathbf{F}$ or $\mathbf{I}_{t}$ we obtain the same, because $\mathbf{F Q}=\mathbf{Q}$, giving $\mathbf{Q}^{\prime} \mathbf{F} \mathbf{Q}=\mathbf{Q}^{\prime} \mathbf{Q}$.

THEOREM 3.2.3. Under the assumptions of Theorem 3.2.1 the following form of $g$-inverse $\mathbf{F}^{-}$can be obtained:

$$
\mathbf{F}_{t t}^{-}=\left[\begin{array}{cc}
\mathbf{C}_{t=f, t-f}^{-1} & \mathbf{0} \\
\mathbf{0} & \mathbf{0}, f \\
f, t-f & f f
\end{array}\right]
$$

where $t-f=h$ and

$$
\left\{\begin{array}{l}
\mathbf{C}^{-1}=\mathbf{I}_{f} \otimes\left(\mathbf{I}_{k-1}+\mathbf{J}_{k-1}\right)=\mathbf{I}_{f} \otimes\left(\mathbf{A}_{1}^{*}\right)^{-1}, \\
\mathbf{A}_{1}^{*}=\mathbf{I}_{k-1}-\frac{1}{k} \mathbf{J}_{k-1} .
\end{array}\right.
$$

Proof. By (3.2.3) the matrix $\mathbf{F}$ can be represented in the form

$$
\mathbf{F}=\mathbf{I}_{f} \otimes \mathbf{A}_{1} .
$$

Let us note that the diagonal and off-diagonal elements of the matrix $\mathbf{A}_{1}$ are equal to $1-1 / k$ and $-1 / k$, respectively.

Let us omit in $\mathbf{A}_{1}$ the last row and the last column to get the matrix

$$
\mathbf{A}_{1}^{*}=\mathbf{I}_{k-1}-\frac{1}{k} \mathbf{J}_{k-1} .
$$

We have

$$
\mathbf{C}=\mathbf{I}_{f} \otimes \mathbf{A}_{1}^{*}
$$

and $\operatorname{rank}(\mathbf{C})=\operatorname{rank}\left(\mathbf{I}_{f}\right) \operatorname{rank}\left(\mathbf{A}_{1}^{*}\right)=f \cdot(k-1)=f k-f=t-f=h$. Since $\operatorname{rank}(\mathbf{F})=h$ we can apply Lemma 4.1. Then

$$
\mathbf{C}^{-1}=\left(\mathbf{I}_{f} \otimes \mathbf{A}_{1}^{*}\right)^{-1}=\mathbf{I}_{f} \otimes\left(\mathbf{A}_{1}^{*}\right)^{-1}=\mathbf{I}_{f} \otimes\left(\mathbf{I}_{k-1}+\mathbf{J}_{k-1}\right)
$$

since by Lemma 4.2 we have

$$
\left(\mathbf{A}_{1}^{*}\right)^{-1}=\left(\mathbf{I}_{k-1}-\frac{1}{k} \mathbf{J}_{k-1}\right)^{-1}=\mathbf{I}_{k-1}+\mathbf{J}_{k-1} .
$$


REMARK 3.2.1. By using (3.2.8) we get the following formula for $S S_{\mathrm{tr} \text { adj }}$ :

$$
\begin{aligned}
& S S_{\text {tr adj }}=\mathbf{Q}^{\prime} \boldsymbol{\Omega}_{p} \mathbf{Q}=\frac{1}{r} \mathbf{Q}_{1 b}^{\prime}\left[\begin{array}{cc}
\mathbf{I}_{f} \otimes\left(\mathbf{I}_{k-1}+\mathbf{J}_{k-1}\right) & \mathbf{0} \\
\mathbf{0} & \mathbf{0}, f \\
f, t-f & f f
\end{array}\right] \mathbf{Q}
\end{aligned}
$$

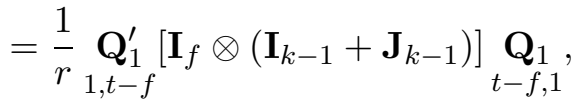

where

$$
\underset{t 1}{\mathbf{Q}}=\left[\begin{array}{c}
\mathbf{Q}_{1} \\
t-f, 1 \\
\mathbf{Q}_{2} \\
f, 1
\end{array}\right]
$$

$\mathbf{Q}_{1}$ corresponds to treatments except those with numbers $k, 2 k, \ldots, f k$ in $\mathbf{Q}$ and

$$
\boldsymbol{\Omega}_{p}=\frac{1}{r} \mathbf{F}^{-}=\frac{1}{r}\left[\begin{array}{cc}
\mathbf{C}^{-1} & \mathbf{0} \\
\mathbf{0} & \mathbf{0}
\end{array}\right]=\frac{1}{r}\left[\begin{array}{cc}
\mathbf{I}_{f} \otimes\left(\mathbf{I}_{k-1}+\mathbf{J}_{k-1}\right) & \mathbf{0} \\
\mathbf{0} & \mathbf{0}
\end{array}\right] .
$$

THEOREM 3.2.2. Under the assumptions of Theorem 3.2.1 the treatment sum of squares adjusted for blocks $S S_{\text {tr adj }}$ can be represented in the following special form:

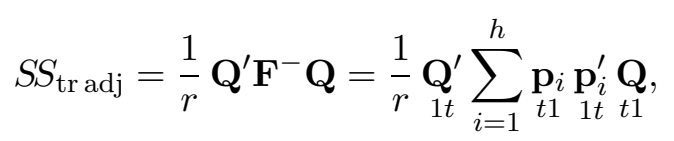

where $\mathbf{p}_{11}, \ldots, \mathbf{p}_{\text {t1 }}$ are $h$ orthonormal eigenvectors of the matrix $\mathbf{F}$ corresponding to the eigenvalue 1 , with multiplicity $h$, of the matrix $\underset{t t}{\mathbf{F}}$, i.e., t-component vectors satisfying the conditions

$$
\begin{aligned}
\mathbf{F} \mathbf{p}_{i}=\mathbf{p}_{i}, \quad \mathbf{p}_{i}^{\prime} \mathbf{p}_{i^{\prime}}=\delta_{i i^{\prime}} & \left(\delta_{i j} \text { is the Kronecker delta }\right) \\
& \text { for } i, i^{\prime}=1,2, \ldots, h, \text { where } h=t-f .
\end{aligned}
$$

Proof. Since $\mathbf{F}^{-}=\mathbf{F}$ by using Theorem 3.2.2, $S S_{\text {tradj }}=\mathbf{Q}^{\prime} \boldsymbol{\Omega}_{p} \boldsymbol{\Omega}=$ $\frac{1}{r} \mathbf{Q}^{\prime} \mathbf{F}^{-} \mathbf{Q}=\frac{1}{r} \mathbf{Q}^{\prime} \mathbf{F} \mathbf{Q}$. The spectral decomposition of the matrix $\mathbf{F}$ in the case of the design with completely confounded interaction because of eigenvalues $\varepsilon_{1}=\cdots=\varepsilon_{h}=1$ is given by $\mathbf{F}=\mathbf{F}^{\prime}=\sum_{i=1}^{h} \varepsilon_{i} \mathbf{p}_{i} \mathbf{p}_{i}^{\prime}=\sum_{i=1}^{h} \mathbf{p}_{i} \mathbf{p}_{i}^{\prime}$, where $\mathbf{F} \mathbf{p}_{i}=\varepsilon_{i} \mathbf{p}_{i}, \mathbf{p}_{i}^{\prime} \mathbf{p}_{i}=1, \mathbf{p}_{i}^{\prime} \mathbf{p}_{j}=0(i \neq j)$.

Now we prove (3.2.15). Since $\underset{t t}{\mathbf{F}} \mathbf{p}_{i}=\varepsilon_{i} \mathbf{p}_{i}$, with eigenvalues $\varepsilon_{i}=1, i=$ $1, \ldots, h$, we have

$$
\mathbf{F} \mathbf{p}_{i}=\mathbf{p}_{i}, \quad\left(\mathbf{I}_{t}-\underset{t t}{\mathbf{F}}\right) \underset{t 1}{\mathbf{p}_{i}}=\mathbf{0}
$$

so that

$$
\underset{t t}{\mathbf{M}} \mathbf{p}_{t}=\underset{t 1}{\mathbf{0}} .
$$

Since $\mathbf{M}$ is as in (3.2.5) we get

$$
\frac{1}{r k}\left(\mathbf{I}_{f} \otimes r \mathbf{J}_{k}\right) \underset{t 1}{\mathbf{p}_{i}}=\underset{t 1}{\mathbf{0}}, \quad \text { i.e. } \quad\left(\mathbf{I}_{f} \otimes \mathbf{J}_{k}\right) \underset{t 1}{\mathbf{p}_{i}}=\underset{t 1}{\mathbf{0}},
$$


and

$$
\underbrace{\left[\begin{array}{ccc}
\mathbf{J}_{k} & & 0 \\
& \ddots & \\
0 & & \mathbf{J}_{k}
\end{array}\right]}_{f \text { columns }}\left[\begin{array}{c}
\mathbf{p}_{I i} \\
k 1 \\
\mathbf{p}_{l I i} \\
k 1 \\
\ldots \\
\mathbf{p}_{f i} \\
k 1
\end{array}\right]=\underset{t 1}{\mathbf{0}}, \quad \mathbf{p}_{t 1}=\underbrace{\left(\mathbf{p}_{I i}, \ldots, \mathbf{p}_{f i}\right)^{\prime}}_{f \text { times }}=\left(\mathbf{p}_{I i}^{\prime}, \ldots, \mathbf{p}_{f i}^{\prime}\right)^{\prime},
$$

with

$$
\underset{k 1}{\mathbf{p}_{I i}}=\left[\begin{array}{c}
p_{1 i} \\
\vdots \\
p_{k i}
\end{array}\right], \quad \mathbf{p}_{k 1}=\left[\begin{array}{c}
p_{(k+1), i} \\
\vdots \\
p_{2 k, i}
\end{array}\right], \ldots, \mathbf{p}_{k 1}=\left[\begin{array}{c}
p_{k(f-1)+1, i} \\
\vdots \\
p_{t, i}
\end{array}\right]
$$

for $i=1, \ldots, h$ and $t=f k$. Hence we obtain (3.2.16).

To solve equation (3.2.16) it is enough to find $h$ orthonormal eigenvectors $\mathbf{p}_{1}, \ldots, \mathbf{p}_{h}$ of the matrix $\mathbf{F}$.

REMARK 3.2.2. Formula (3.2.14) expresses the partition of the $S S_{\text {tradj }}$ in ANOVA into $h$ sums of squares with one degree of freedom each.

The treatment contrasts $\sum_{i=1}^{k} l_{i j m} Q_{i j m}, \sum_{i=1}^{k} l_{i j m}=0(j=1, \ldots, k-1$; $m=1, \ldots, f), t=f k$, have one degree of freedom each as in the following formula:

$$
S S_{\text {tr adj }}=\sum_{m=1}^{f} \sum_{j=1}^{k-1} \frac{\left(\sum_{i=1}^{k} l_{i j m} Q_{i j m}\right)^{2}}{r \sum_{i=1}^{k} l_{i j m}^{2}}
$$

with $h=f(k-1)=t-f$ degrees of freedom, where $Q_{i j m}$ are treatment adjusted totals; the indices $i, j, m$ in $l_{i j m}$ denote: $i$-number of coefficients in the contrast, $j$-number of contrasts in the block, and $m$-number of blocks.

In intra-block ANOVA we have $\sum_{j=1}^{k} l_{j m} Q_{j m}=\sum_{j=1}^{k} l_{j m}\left(T_{j m}-C_{m}\right)=$ $\sum_{j=1}^{k} l_{j m} T_{j m}$, since $Q_{j m}=T_{j m}-C_{m}$, where $C_{m}=$ const within blocks for $m=1, \ldots, f$.

It may be instructive to write

$$
\left.S S_{\text {tr adj }}=\frac{1}{r} \sum_{i=1}^{h}\left(\mathbf{p}_{i}^{\prime} \mathbf{Q}\right)^{2}=r \sum_{i=1}^{h} \widehat{\left(\mathbf{p}_{i}^{\prime} \boldsymbol{\tau}\right.}\right)^{2},
$$

where $\widehat{\mathbf{p}_{i}^{\prime} \boldsymbol{\tau}}$ is the best linear unbiased estimator (BLUE) of the contrast $\mathbf{p}_{i}^{\prime} \boldsymbol{\tau}$.

The analysis of variance for testing treatment effects may be given in the following table: 
Table 1. ANOVA of the completely confounded component of three-factor interaction of the factorial experiment

\begin{tabular}{|c|c|c|c|}
\hline Source & D.F. & $S S$ & M.S. \\
\hline $\begin{array}{c}\text { Block (ignoring } \\
\text { treatments) }\end{array}$ & $r f-1$ & $S S_{\mathrm{bl}}$ & - \\
\hline Treatments & $\begin{array}{c}t-f \\
=\operatorname{rank} \mathbf{F}\end{array}$ & $\begin{array}{c}S S_{\mathrm{tr} \text { adj }} \\
=\frac{1}{r} \mathbf{Q}^{\prime} \mathbf{F} \mathbf{Q} \\
1 t \\
\begin{array}{c}1 t \\
\mathbf{Q}^{\prime} \sum_{i=1}^{h} \mathbf{p}_{i} \mathbf{p}_{i}^{\prime} \mathbf{Q} \\
1 t\end{array}\end{array}$ & $\frac{S S_{\text {tr adj }}}{h}$ \\
\hline Residual & $f(k-1)(r-1)=\nu_{e}$ & $S S_{e}=\mathbf{y}^{\prime} \psi \mathbf{y}$ & $\frac{S S_{e}}{\nu_{e}}$ \\
\hline Total & $n-1$ & $S S_{y}=\sum_{i=1}^{n} y_{i}^{2}-C_{y}$ & - \\
\hline
\end{tabular}

Here $S S_{b l}=\frac{1}{k} \sum_{j=1}^{b} B_{j}^{2}-C_{y}, C_{y}=\frac{1}{n}\left(\sum_{i=1}^{n} y_{i}\right)^{2} . S S_{\text {tr adj }}$ can be written as $\frac{1}{r} \sum_{i=1}^{h}\left(\mathbf{p}_{i}^{\prime} \mathbf{Q}\right)^{2}$.

Note that $S S_{e}=\mathbf{y}^{\prime} \boldsymbol{\phi} \mathbf{y}-S S_{\text {tr adj }}=\mathbf{y}^{\prime} \boldsymbol{\psi} \mathbf{y}$, where $\boldsymbol{\psi}=\boldsymbol{\phi}-\boldsymbol{\phi} \boldsymbol{\Delta}^{\prime} \mathbf{A}^{-} \boldsymbol{\Delta} \boldsymbol{\phi}$ (see, e.g., Caliński and Kageyama, 2000, p. 65). The test function for the hypothesis $\tau_{1}=\tau_{2}=\cdots=\tau_{t}$ is $F=\frac{S S_{\mathrm{tr} \text { adj }}}{h}: \frac{S S_{e}}{\nu_{e}}$ with $h$ and $\nu_{e}$ degrees of freedom. In the case of $D(t, b, k, r)=D\left(4^{3}, 12,16,3\right)$ and $f=4$ we have $h=4^{3}-4=60$ and $\nu_{e}=f(k-1)(r-1)=4(16-1)(3-1)=120$ degrees of freedom. The total number of observations is $n=r t=3 \cdot 4^{3}=192$ (number of plots in the field experiment, Oktaba, 1956; Oktaba et al., 1956). Further remarks are given in Sections 3.3 and 3.4.

Table 1 is general for any case of an orthogonal disconnected block design, where $f-1$ contrasts are completely confounded with blocks (not necessarily contrasts concerning interaction effects).

3.3. The graphical method "O" of constructing sets of orthonormal eigenvectors of the matrix $\mathbf{F}$ in the disconnected fixed design $D(t, b, k, r)$ with completely confounded interaction/main effect. The method of constructing sets of mutually orthogonal contrasts which we called the graphical method "O" was given by Oktaba (1958). It is based upon the theorem of number theory concerning the systematic expansion of natural numbers to any natural numerical base (Sierpiński, 1925). This is the method of linking $t$ points to the base (natural number) $a \geq 2$. The graphical configuration obtained from performing conjunctions is uniquely determined by the expansion of natural numbers. This method can be used in ANOVA and specially in ANOVA of a disconnected fixed design $D(t, b, k, r)$ with completely confounded interaction. This is stated in Theorem 3.3.1. It also presents constructing an orthonormal matrix $\mathbf{F}$ which can be found directly from the configuration of conjunctions in the graph. 
THEOREM 3.3.1. Under the assumptions of Theorem 3.2.1 the sum $\sum_{i=1}^{h} \mathbf{p}_{i} \mathbf{p}_{i}^{\prime}$, which is as in formula (3.2.14) can be found by the graphical method "O".

Proof. From Theorem 3.2.4 by (3.2.18), (3.2.10) and (3.2.11) we can use the graphical method "O" with respect to each of the $f$ blocks of size $k$, where $t=f k, h=t-f=\operatorname{rank}\left(\mathbf{F}^{-}\right)$. For each of the $f$ blocks we get $k-1$ independent contrasts, so we obtain $h=f(k-1)=t-f$ orthonormal eigenvectors corresponding to $h$ eigenvalues $\varepsilon=1$ with multiplicity $h$. These eigenvectors fulfill conditions (3.2.15), as it should be. The same graph can be constructed for each of the $f$ incomplete blocks.

REMARK 3.3.1. It is evident that the graphical method can be replaced by any other method. But this one seems to be very simple and natural. The choice of method depends on the problem in which the experimenter is interested.

4. Appendix. Some matrix results. The main results required for this paper are given in this Appendix. They are stated without proof as they are straightforward and can be found in most books on statistics (e.g. Rao, 1973).

LEMmA 4.1. If $\operatorname{rank}(\mathbf{A})=\operatorname{rank}(\mathbf{C})=r$ and $\mathbf{A}=\left[\begin{array}{cc}\mathbf{C} & \mathbf{D} \\ \mathbf{E} & \mathbf{F}\end{array}\right]$, then

$$
\mathbf{A}^{-}=\left[\begin{array}{cc}
\mathbf{C}^{-1} & \mathbf{0} \\
\mathbf{0} & \mathbf{0}
\end{array}\right]
$$

where $\operatorname{rank}(\mathbf{A})=\operatorname{rank}\left(\mathbf{A}^{-}\right)$and $\mathbf{A}^{-} \mathbf{A} \mathbf{A}^{-}=\mathbf{A}^{-}$.

LEMma 4.2 (Rao, 1973, p. 67). We have

$$
\left|\mathbf{Z}_{a, b}\right|=\left|\begin{array}{cccc}
a & b & \ldots & b \\
b & a & \ldots & b \\
\ldots & \ldots & \ldots & \ldots \\
b & b & \ldots & a
\end{array}\right|=(a-b)^{k-1}[a+(k-1) b]
$$

where $\mathbf{Z}_{a, b}$ is a $k \times k$ matrix. Hence:

$$
\text { If } a=1-\frac{1}{k}, b=-\frac{1}{k}, \quad \text { then } \quad\left|\mathbf{Z}_{a, b}\right|=0 \text {. }
$$

$$
\text { If } b=a-1, \text { then } \begin{aligned}
\left|\mathbf{Z}_{a, a-1}\right| & =\left|\begin{array}{cccc}
a & a-1 & \ldots & a-1 \\
a-1 & a & \ldots & a-1 \\
\ldots \ldots & \ldots & \ldots & \ldots \\
a-1 & a-1 & \ldots & a
\end{array}\right| \\
& =k(a-1)+1=\left|\mathbf{I}_{k}+(a-1) \mathbf{J}_{k}\right| .
\end{aligned}
$$


Lemma 4.3. For $k=2,3,4, \ldots$ the g-inverse $\mathbf{A}_{1}^{-}$of the matrix

$$
\mathbf{A}_{1}=\mathbf{I}_{k}-\frac{1}{k} \mathbf{J}_{k}
$$

is of the form

$$
\left\{\begin{array}{l}
\mathbf{A}_{1}^{-}=\left(\mathbf{I}_{k}-\frac{1}{k} \mathbf{J}_{k}\right)^{-}=\left[\begin{array}{cccc}
1-\frac{1}{k} & -\frac{1}{k} & \ldots & -\frac{1}{k} \\
-\frac{1}{k} & 1-\frac{1}{k} & \ldots & -\frac{1}{k} \\
-\frac{1}{k} & -\frac{1}{k} & \ldots & 1-\frac{1}{k}
\end{array}\right]=\mathbf{A}_{1}=\mathbf{A}_{1}^{2} \\
\operatorname{trace}\left(\mathbf{A}_{1}\right)=\operatorname{rank}\left(\mathbf{A}_{1}\right)
\end{array}\right.
$$

LEMMA 4.4.

$$
\left(\mathbf{I}_{k-1}-\frac{1}{k} \mathbf{J}_{k-1}\right)^{-1}=\mathbf{I}_{k-1}+\mathbf{J}_{k-1}
$$

where $\mathbf{I}_{k}$ is the identity matrix, and $\mathbf{J}_{k-1}$ the matrix with all elements equal to 1.

Acknowledgements. The author thanks the referees for helpful comments that improved the presentation of this paper.

\section{References}

R. C. Bose and W. S. Connor (1952), Combinatorial properties of group divisible incomplete block designs, Ann. Math. Statist. 23, 367-383.

R. C. Bose and K. Kishen (1946), On the problem of confounding in the general symmetrical factorial design, Sankhyā 5, 21-36.

T. Caliński (1983), A General Theory of Intrablock Analysis, Department of Math. and Statist. Method., Academy of Agriculture, Poznań, 1-38 (mimeographed notes).

T. Caliński, B. Ceranka and S. Mejza (1979), Efficiency of a block design, IX Methodological Agro-biometrical Colloquium, Vol. I, 178-189, Polish Academy of Sciences, Warszawa (in Polish).

T. Caliński and S. Kageyama (2000), Block Designs. A Randomization Approach, Vol. I. Analysis, Springer, New York.

T. Caliński and S. Kageyama (2003), Block Designs. A Randomization Approach, Vol. II. Design, Springer, New York.

W. G. Chochran and G. M. Cox (1950), Experimental Designs, Wiley, New York.

W. S. Connor and W. H. Clatworthy (1954), Some theorem for partially balanced designs, Ann. Math. Statist. 85, 100-112.

D. J. Finney (1947), The construction of confounded arrangements, Emp. J. Exp. Agr. $15,107-112$.

R. A. Fisher (1942), The theory of confounding in factorial experiments in relation to the theory of groups, Ann. Eugenics 11, 341-353.

P. W. M. John (1980), Incomplete Block Designs, Dekker, New York.

R. M. Jones (1959), On a property of incomplete blocks, J. Roy. Statist. Soc. Ser. B 21, $172-179$.

O. Kempthorne (1952), The Design and Analysis of Experiments, Wiley, New York. 
K. R. Nair (1938), On a method of getting confounded arrangement in the general symmetrical type of experiments, Sakhyā 4, 121-138.

A. K. Nigam, P. D. Puri and V. K. Gupta (1988), Characterizations and Analysis of Block Designs, Wiley Eastern Limited, New Delhi.

W. Oktaba (1956), Method of complete confounding of interactions with subblocks in a factorial experiment with two, three, four and five levels of each factor, Ann. Univ. Mariae Curie-Skłodowska 11, 123-186 (in Polish).

W. Oktaba (1958), On certain method of constructing sets of mutually orthogonal comparisons, ibid. $12,1,5-22$.

W. Oktaba (1970), Theory of Experimental Designs, PAN, Warszawa (in Polish).

W. Oktaba (1971), Methods of Mathematical Statistics in Experimentation, PWN, Warszawa (in Polish).

W. Oktaba (2002), History of the Theory of Experiment, Lublin Scientific Society, Lublin (in Polish).

W. Oktaba, W. Rejmak and M. Warteresiewicz (1956), Efficiency of confounded design and its use in the investigations on the influence of sowing density and nitrogen dressing levels on the yield of four varieties of spring-sown wheat, ibid. 11, 187-226 (in Polish).

S. C. Pearce (1963), The use and classification of non-orthogonal designs, J. Roy. Statist. Soc. Ser. A 126, 353-377.

S. C. Pearce (1970), The efficiency of block design in general, Biometrika 57, 339-346.

S. C. Pearce (1976), Concurrences and quasi-replication. An alternative approach to precision in designed experiments, Biom. J. 18, 105-116.

S. C. Pearce (1983), The Agricultural Field Experiment: A Statistical Examination of Theory and Practice, Wiley, Chichester.

S. C. Pearce, T. Caliński and T. F. de C. Marshall (1974), The basic contrasts of an experimental design with special reference to the analysis of data, Biometrika 61, 449460.

C. R. Rao (1951), A theorem in least squares, Sankhyā 11, 9-12.

C. R. Rao (1973), Linear Statistical Inference and Its Applications, Wiley, New York.

H. Scheffé (1959), The Analysis of Variance, Wiley, New York.

W. Sierpiński (1925), Theory of Numbers, Warszawa (in Polish).

A. P. Street and D. J. Street (1987), Combinatorics of Experimental Design, Clarendon Press, Oxford.

K. D. Tocher (1952), The design and analysis of block experiments, J. Roy. Statist. Soc. Ser. B 14, 45-100.

F. Yates (1933), The principles of orthogonality and confounding in replicated experiments, J. Agric. Sci. 23, 108-145.

F. Yates (1937), The design and analysis of factorial experiments, Technical Comm. 35, Imp. Bureau of Soil Science.

Department of Mathematical Statistics

Institute of Applied Mathematics

Agricultural University

Akademicka 13

20-934 Lublin, Poland

E-mail: sek314@ursus.ar.lublin.pl

Received on 23.2.2002;

revised version on 5.10.2004 